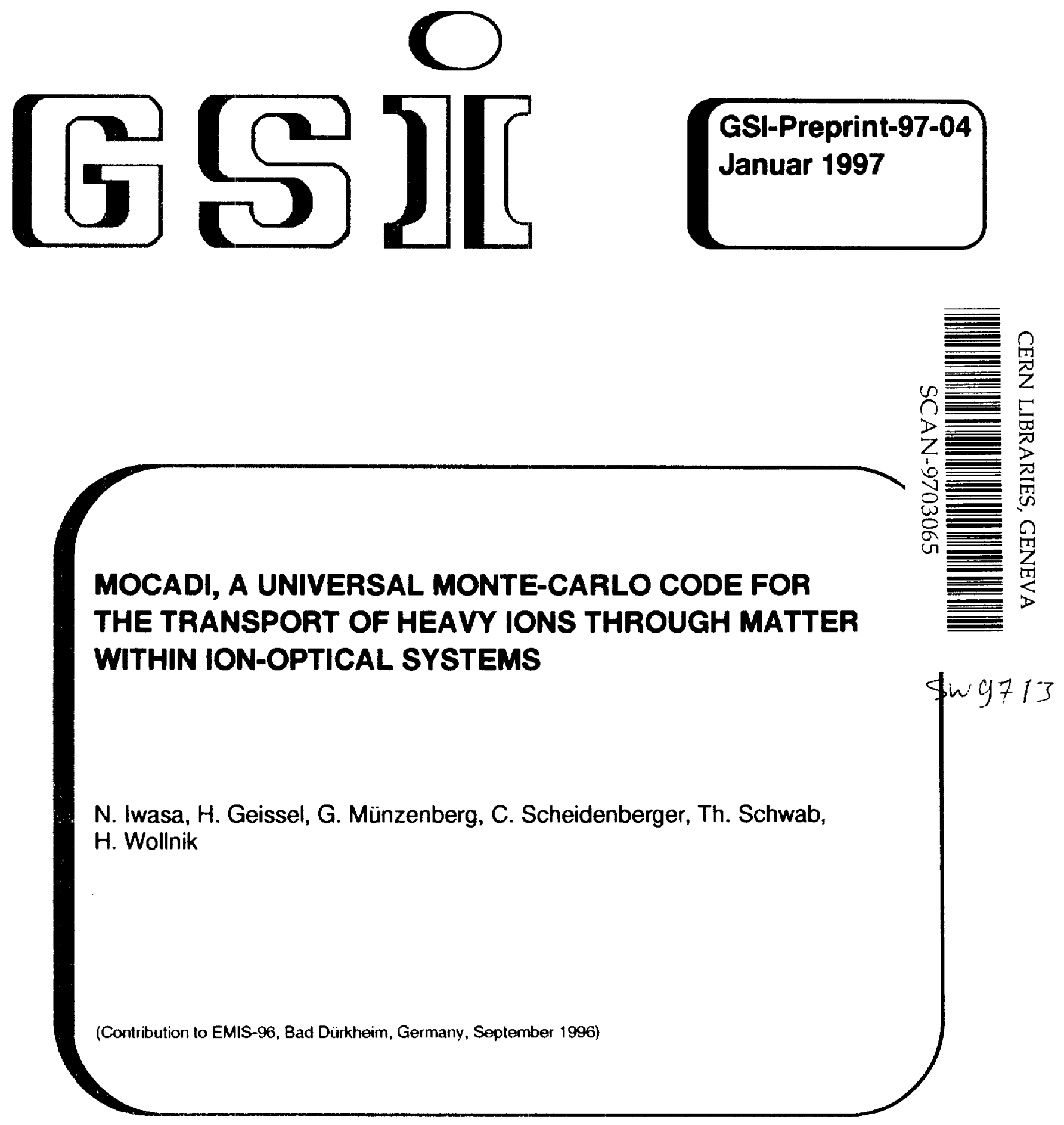

Gesellschaft für Schwerionenforschung $\mathrm{mbH}$ Planckstraße 1 - D-64291 Darmstadt - Germany Postfach 110552 - D-64220 Darmstadt • Germany 


\title{
MOCADI, a universal Monte-Carlo code for the transport of heavy ions through matter within ion-optical systems
}

\author{
N. Iwasa ${ }^{a, 1}, H$. Geissel $^{a}$, G. Münzenberg ${ }^{a}$, C. Scheidenberger ${ }^{a}$, \\ Th. Schwab ${ }^{a, b}$, H. Wollnik ${ }^{b}$ \\ a GSI, D-64291 Darmstadt, Germany \\ ${ }^{\circ}$ Univ. Gießen, D-35392 Gießen, Germany
}

\begin{abstract}
A precise knowledge of the atomic and nuclear interactions of ions penetrating through matter placed within ion-optical systems is important for experiments with energetic secondary nuclear beams as well as for applications. For this purpose the code MOCADI was developed, incorporating both the higher-order ion-optical treatment and the atomic and nuclear interactions of heavy ions. MOCADI was used for the design of the projectile fragment separator FRS and for the preparation of the experimental programme. At that time only scarce information was available on the interaction of relativistic heavy ions penetrating thick layers of matter. In the meantime, new experimental results and refined theories have contributed to improve the theoretical descriptions used in the code. This progress and the general structure and features of the programme are outlined in this contribution.
\end{abstract}

Keywords:

PACS: XXXX EMIS-13; NIM-B; proceedings; LAT $\mathrm{E} X$;

\section{Introduction}

A precise knowledge of the atomic and nuclear interactions of heavy ions with matter is crucial for various nuclear physics experiments and applications using secondary nuclear beams. A detailed understanding of the physical mechanisms for the production, separation, and atomic and nuclear interaction with matter is required. Moreover, it is necessary to combine the knowledge

\footnotetext{
${ }^{1}$ Corresponding author. Tel. +49 615971 2665, fax +49 615971 2902, e-mail N.Iwasa@gsi.de
} 
of the interaction of swift heavy ions in matter with the transport properties of ion-optical systems.

There are many codes which calculate the transport of ions through ion-optical systems such as COSY [1] GICO [2], MIRKO [3] or TRANSPORT [4]. The purpose of the code MOCADI [5] is to transport an ensemble of heavy ions through systems which consist of ion-optical elements and matter, i. e., to include non-Liouvillian optics [6]. Since non-Liouvillian processes generally affect all particle coordinates, a proper treatment of the complete phase space is required. In special cases an analytical program using only the dispersive coordinate and results of first-order optics can be valuable [7]. The codes LISE [8] and INTENSITY [9] applied at medium-energy fragmentation facilities are based on first-order optics and use Gaussian-convolution techniques.

The Monte-Carlo program MOCADI was the first code capable of tracing an arbitrary phase-space-density distribution of relativistic heavy ions through ion-optical systems taking into account all particle coordinates and higherorder image aberrations as well as atomic and nuclear interaction with matter. It was developed in the late 80's [5] for design studies of the GSI fragment separator FRS [10]. It has been used for the preparation of the experimental programme at the FRS and its combination with the storage and cooler ring ESR [11]. MOCADI is now routinely used for preparation and analysis of experiments with secondary nuclear beams: e. g. for rate estimations, for studying beam properties, separation quality, implantation profiles, optimization of the experimental setup, and transmission studies. It has proven to be an excellent tool for these purposes and all necessary inputs concerning ionoptics and reactions are steadily improved according to experimental results. Some of them will be presented in the forthcoming section.

\section{Interaction of heavy ions with matter}

When an energetic ion, characterized by its atomic number $Z_{p}$, mass number $A_{p}$, and ionic charge state $q$, penetrates through matter, different atomic and nuclear processes can take place. The particle

- can change its ionic charge state by capturing or losing electrons,

- can capture or lose nucleons in nuclear reactions (e. g. charge-changing reactions, fission, fragmentation, electromagnetic dissociation),

- will change its kinetic energy and direction as a result of both nuclear and atomic collisions.

All these processes change the longitudinal and transverse phase-space density and in case of nuclear interactions also the particle identity. 


\subsection{Nuclear interactions}

Projectile fragments are produced in peripheral nuclear collisions: impact parameters smaller than the sum of the radii of the colliding nuclei lead to abrasion of nucleons, larger impact parameters lead to Coulomb excitation [12]. In both cases the excited nucleus evaporates particles or may fission.

Production cross sections for projectile fragmentation can be well estimated using the semi-empirical parametrizations (e. g. EPAX [13]), intranuclearcascade calculations (e. g. ISAPACE [14]), or abrasion-ablation calculations (e. g. ABRABLA [15]). As a representative example figure 1 shows the predictions of the three mentioned codes in comparison with measured data for $\mathrm{Zn}$ fragments produced via fragmentation of $500 \mathrm{~A} \mathrm{MeV}{ }^{86} \mathrm{Kr}$ in a beryllium target [16]. The generally good agreement shows that the physical processes leading to the fragment isotopic distribution are properly described by the different models. The nuclear absorption can be described by the total reaction cross section [17].

The longitudinal momentum distribution of a specific fragment can be determined from the mean momentum loss in the reaction [18] and from the momentum spread. The Fermi momenta of the abraded nucleons determine the width of the prefragment-momentum distribution [19]. In general the evaporation of nucleons additionally contributes to the momentum distribution of the measured reaction products. This case is taken into account by the systematics of Morrissey [20] using experimental data. A comparison of representative experimental data with the predictions of refs. [19] and [20] is shown in the lower part of figure 1.

Fission of relativistic uranium nuclei has proven to be an excellent source for providing beams of medium-heavy neutron-rich nuclei [21]. The kinematics of the relativistic fission products is determined from the total kinetic energy of the fission process transformed into the laboratory frame [22].

The nuclear interaction is implemented in MOCADI in terms of cross sections $[12,13,17]$ and reaction kinematics $[18-20,22]$.

\subsection{Atomic interactions}

A precise knowledge of the atomic interactions in matter is especially important for efficient in-flight separation of energetic fragments using thick degraders placed at dispersive focal planes ( $B \rho-\Delta E-B \rho$ separation-method) [23]. Energy loss, energy-loss straggling, charge-state population, and multiple angular scattering are key parameters to characterize the results of the atomic 

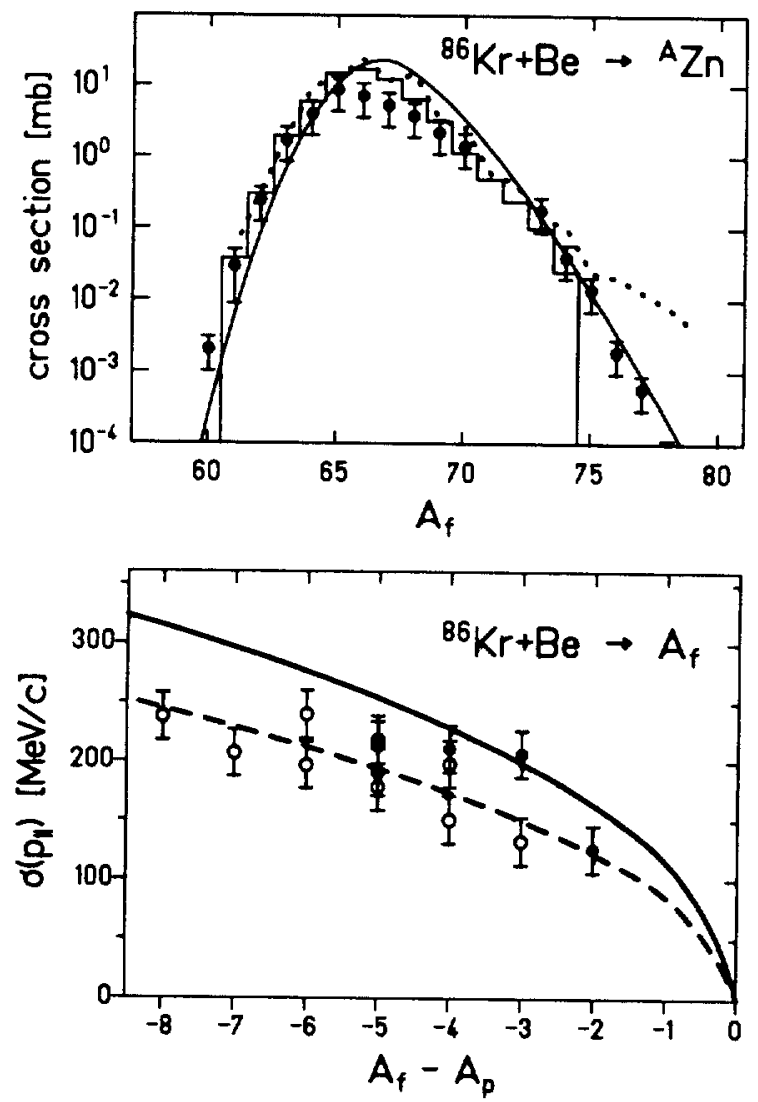

Fig. 1. Upper part: Experimentally determined projectile fragmentation cross sections for $\mathrm{Zn}$ isotopes produced in $500 \mathrm{~A} \cdot \mathrm{MeV}{ }^{86} \mathrm{Kr}+\mathrm{Be}$ collisions [16] compared with predictions of EPAX [13] (solid line), ISAPACE [14] (histogram), and ABRABLA [15] (dotted line). Lower part: Measured widths of the longitudinal momentum distribution as a function of the difference of fragment mass $A_{f}$ and projectile mass $A_{p}$ in comparison with the Goldhaber theory [19] (solid line) and the Morrissey systematics [20] (dashed curve). Pure proton-removal channels are represented by full symbols.

collisions in matter, and theoretical descriptions of these observables are implemented in our Monte Carlo programme. Taking into account the practical requirements in high-energy fragmentation experiments we assume that the layers of matter are thick enough to avoid the descriptions of pre-equilibrium effects.

In the following we present recent experimental results on charge-state distributions, stopping powers, energy straggling, and angular scattering of heavy ions compared with the theories used in MOCADI.

A first estimation on the ionic charge states of heavy ions can be obtained by the velocity criterion postulated by N. Bohr [24]: projectile electrons are stripped off in atomic collisions when the projectile velocity exceeds the orbital velocity of the bound electrons. This means that at sufficiently high energies the projectiles are bare or carry only few bound electrons which simplifies a 
stringent comparison with theory. Our experimental results obtained with the FRS illustrate the very good agreement with theoretical predictions [25] for all investigated projectile-target combinations at energies above $200 \mathrm{~A} \cdot \mathrm{MeV}$. Figure 2 shows as an example the equilibrium charge-state distributions of $\mathrm{Xe}$, $\mathrm{Au}$, and $\mathrm{U}$ ions in $\mathrm{Cu}$ targets as a function of the projectile energy.

The mean energy loss and the energy straggling of ions in matter must be known very accurately for selecting the magnetic rigidity of the ion-optical stages following the layers of matter, for the predictions of the transmission properties of the fragments within the optical system, and also for the calculations of the range distributions in special stopping materials. For example, the nuclear reaction products are implanted in detectors to study their decay properties.

The energy loss of relativistic heavy ions mainly results from collisions with target electrons. In a first approximation it can be calculated using the relativistic Bethe formula [27]. However, precise energy loss measurements [28] of very heavy projectiles demonstrate large systematic deviations from the results of theoretical calculations based on the first Born approximation.

Incorporating the relativistic Mott scattering cross section and the Bloch correction [29], the Bethe stopping power theory yields then a perfect agreement with the experimental data as illustrated for representative results in figure 3 .

The energy-loss straggling receives its major contributions from close collisions with target electrons and from statistical fluctuations of the ionic charge states (charge-exchange straggling). Recent experimental results on energy-loss distributions of relativistic projectiles up to uranium [30] demonstrate that the predictions based on the first order perturbation theories underestimate the data by up to a factor of 2.5. However, a rigorous relativistic theory developed by Lindhard and Sørensen [29] can reproduce very well the measured results as indicated in figure 3 . The charge-exchange straggling, treated in a basic description by Sigmund [31], yields a small contribution at $800 \mathrm{~A} \cdot \mathrm{MeV}$ even for the heaviest projectiles.

The angular scattering distribution can severely limit the transmission and resolution properties of spectrometers, especially if thick layers of matter are involved. The Coulomb deflection of ions in the field of the target nucleus can be described by a classical treatment for heavy collision systems at all presently available energies. The simple formula from ref. [32] yields good agreement for the heavy ion data displayed in figure 3 . 


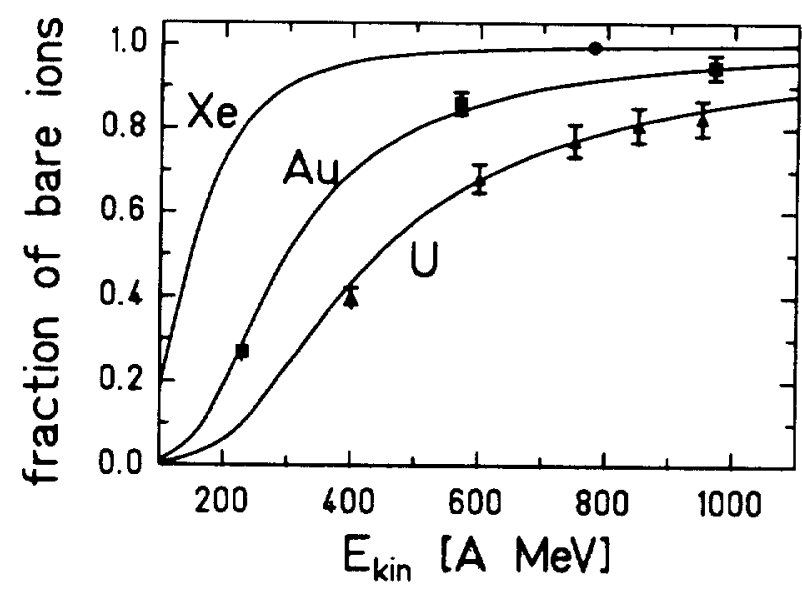

Fig. 2. Calculated and measured fractions of bare ions in charge-state equilibrium for $\mathrm{Xe}, \mathrm{Au}$, and $\mathrm{U}$ projectiles penetrating through copper targets [26] as a function of the projectile energy.

\section{Transport through matter within ion-optical systems}

In ion optics the transfer function images the initial phase space to any desired position in the system. A widely used approximation for the transfer function is represented by the matrix formalism [33]. The matrix elements are the coefficients of expanded Taylor series. The order of the optical description defines to which order the Taylor series is evaluated.

Many ion-optical codes, such as COSY, GICO, MIRKO, Raytrace, and TRANSPORT, can be applied to calculate the transfer matrices of any electromagnetic system. The matrices, obtained from these calculations have been implemented in MOCADI to transform the phase-space coordinates of the ions through any selected part of an optical device. By successive application of this transformation one can easily trace a particle through complex ion-optical systems also if the ions interact with matter placed within the device $[6,5$, and references therein]. The theoretical descriptions mentioned above are applied to modify the coordinates of the particles in case of atomic and nuclear interactions in combination with the established optical imaging. In this way the particles propagate through a system consisting of ion-optical elements and layers of matter.

\section{Structure and applications of the code MOCADI}

The code MOCADI, written in the language $\mathrm{C}$, consists of modules which can be arranged in a desired sequence to realistically simulate a complex exper- 

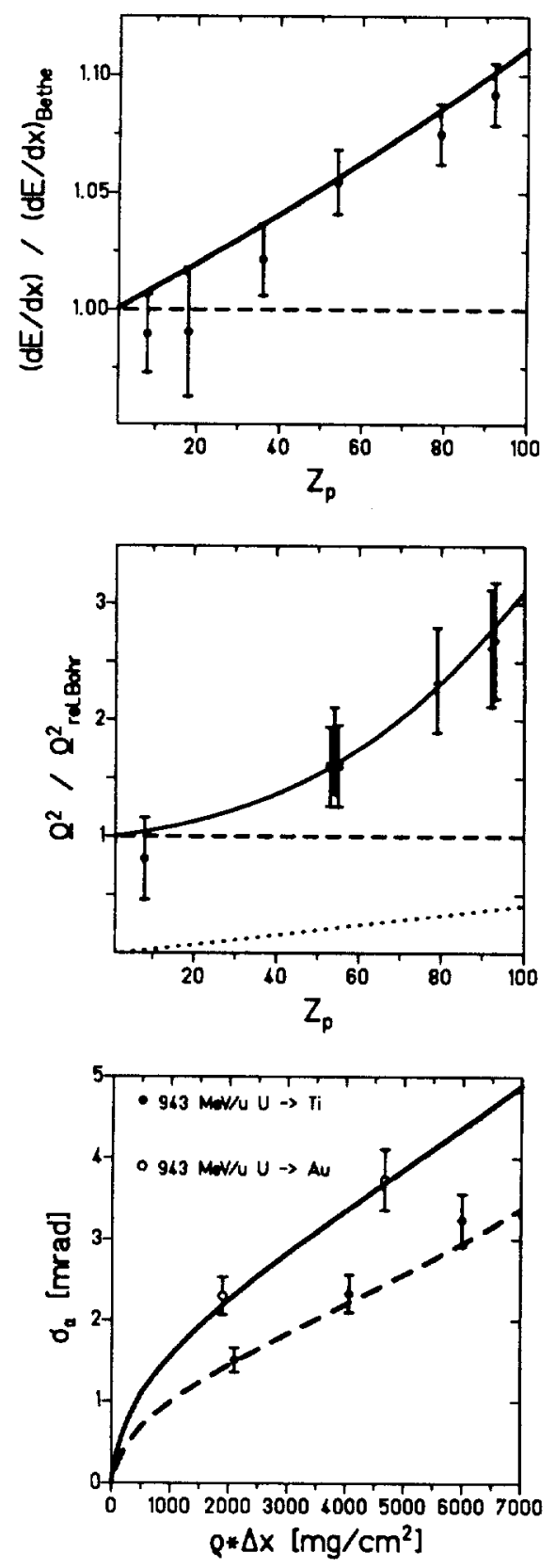

Fig. 3. Representative results of recent atomic-collision studies at the FRS: for heavy nuclei the Bethe and Bohr theories fail since both are based on first-order perturbation calculations applicable only for nuclei with small atomic number. The contribution of charge-exchange straggling is indicated by the dottel line. Predictions for multiple angular scattering [32] are in good agreement with our measured data for $\mathrm{U}$ impinging on $\mathrm{Ti}$ and $\mathrm{Au}$ targets.

imental setup. The initial coordinates can be randomly selected to represent for example the incident projectile beam in front of a target at the entrance of a spectrometer. The atomic and nuclear interactions are simulated in the module 'target'. The particles emerging from the target are then transported through a part of an ion-optical device until the next interaction with mat- 
ter is introduced. The whole ion-optical device is realistically described by the matrix formalism, the drift lengths, the apertures, the geometrical boundaries of vacuum chambers, and slits. The coordinates of the particles can be recorded and stored at any desired position of the device. These data can be treated like experimental list-mode data. Realistic transmission properties of ions analyzed in complex devices can easily be obtained in this way. Specially shaped layers of matter, often called profiled degraders, placed in dispersive focal planes, are important tools to provide spatially separated monoisotopic beams of energetic fragments $[6,10,34,35]$. MOCADI is particularly well suited to simulate the performance of such a device since all the particle coordinates are considered. A representative example is presented in figure 4 . The $1 \mathrm{~A} \cdot \mathrm{GeV}{ }^{238} \mathrm{U}$ projectile beam is used to produce, via projectile fragmentation, the neutron-rich nuclei ${ }^{215} \mathrm{~Pb}$ in a $1 \mathrm{~g} / \mathrm{cm}^{2}$ beryllium target. The FRS optical system is optimized to spatially separate this fragment beam by using the atomic energy loss properties in a $4.6 \mathrm{~g} / \mathrm{cm}^{2}$ aluminum degrader at the central focal plane. The relevant coordinates in the different sections of the setup are displayed in the figure. The spatial coordinate $X$ is in direction of the magnetic dispersion and $X^{\prime}$ represents the corresponding angle. Similarly $Y$ and $Y^{\prime}$ are the coordinate and the angle respectively, in the plane perpendicular to $Y$ and the optical axis. The energy distribution is given in addition to the transverse emittances. In this case the degrader is shaped so as to provide an achromatic image at the final focus of the system, as clearly illustrated in the energy distribution plotted versus $X$.

In many experiments and applications it is necessary to implant the separated radioactive nuclear beams. The range distribution is then strongly dependent on the incident energy distribution of the nuclear beam, see figure 5. Differently shaped degraders placed a dispersive focal plane can drastically influence the range distributions. This is demonstrated in figure 5 with a MOCADI calculation using the parameters of the previous example. The thickness of all degraders along the optical axis is the same but only the shape of the degrader is changed [6], i. e., the slope of the material distribution (wedge angle) in the $X$ direction is varied. In our example the ${ }^{215} \mathrm{~Pb}$ nuclei are implanted in silicon material to simulate a stack of semiconductor detectors. A homogenous degrader (wedge angle equal to zero) yield a range distribution with the largest width whereas the monoenergetic shape provides an implantation profile which approaches the ideal conditions one could obtain if the fragment beam is cooled in a storage ring to a momentum spread of $10^{-6}[36]$.

The spatial isotopic separation of all elements up to the heaviest nuclear beams produced via fragmentation of $950 \mathrm{~A} \cdot \mathrm{MeV}$ uranium projectiles has recently been achieved in experiments with the FRS separator [35]. The comparison of the MOCADI simulation with these experimental results represents a stringent test for the validity of the theoretical models in the code and also for the knowledge of the experimental conditions such as mechanical imperfections 


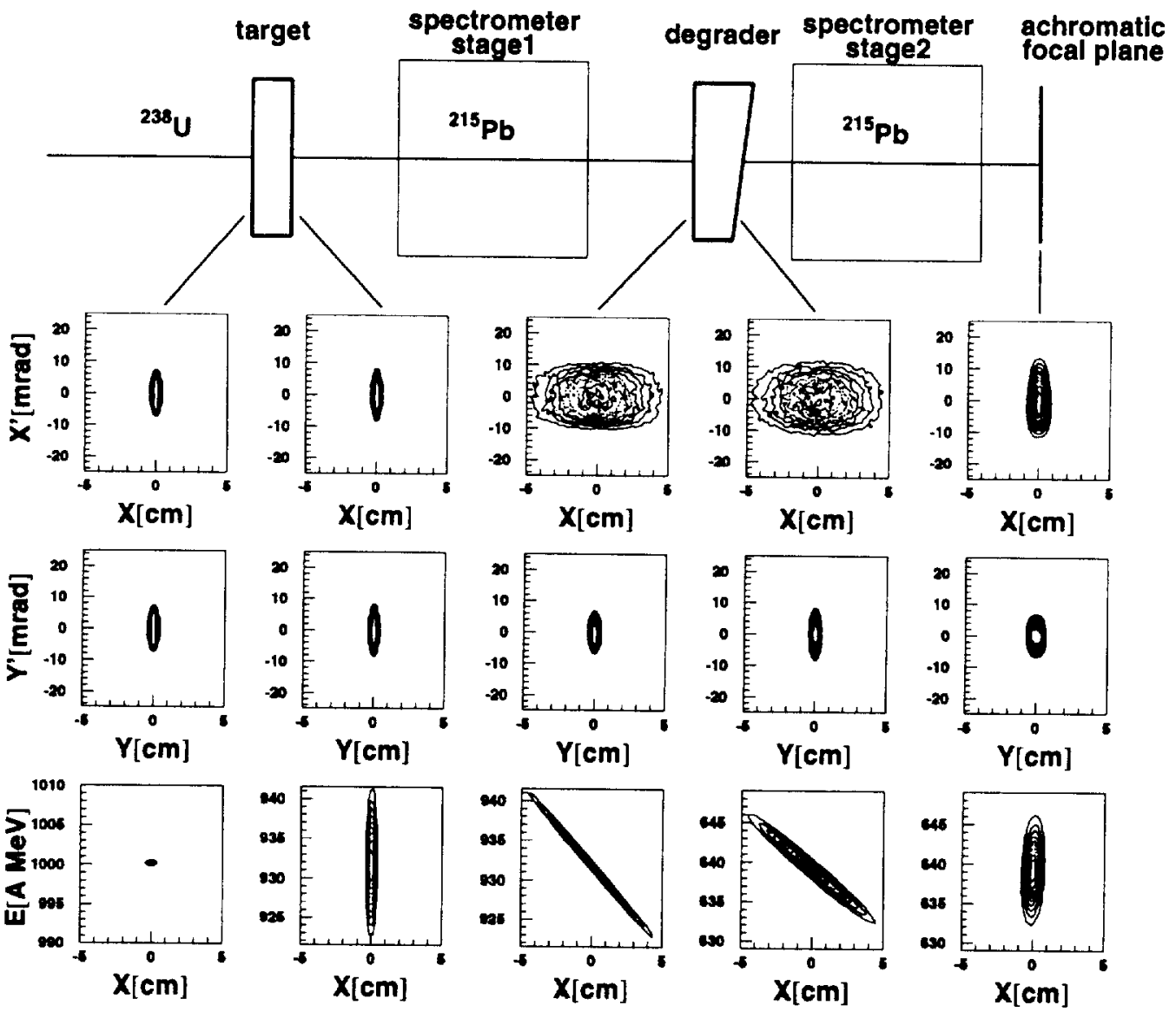

Fig. 4. Calculated transverse emittances and energy-position correlation of ${ }^{215} \mathrm{~Pb}$ ions produced via fragmentation of $1 \mathrm{GeV} / \mathrm{u}^{238} \mathrm{U}$ projectiles in a $1 \mathrm{~g} / \mathrm{cm}^{2}$ beryllium target and slowed down in a $4.6 \mathrm{~g} / \mathrm{cm}^{2}$ aluminum degrader placed at the central focal plane of the fragment separator FRS. The horizontal direction ( $X$-direction) is the dispersive direction. The corresponding schematic experimental setup is shown at the top.

of the degrader, detector performances, and ion-optical image aberrations. In the experiment an achromatic degrader of $5.3 \mathrm{~g} / \mathrm{cm}^{2}$ aluminum was used and the FRS was tuned to separate ${ }^{236} \mathrm{U}$ nuclei. The experimental results are compared with the MOCADI simulation in figure 6 . In general, the agreement between the experimental results and the calculation is very good. The slightly narrower distribution of the MOCADI calculation points to some thickness nonuniformities of the degrader system in the FRS which will be improved.

In summary it can be concluded that MOCADI is a very useful programme especially for planning and analyzing experiments with relativistic heavy ions in complex ion-optical systems with many layers of matter placed at different positions in the device.

It is a pleasure to thank K. Sümmerer and W. Catford for fruitful discussions and for carefully reading the manuscript. 


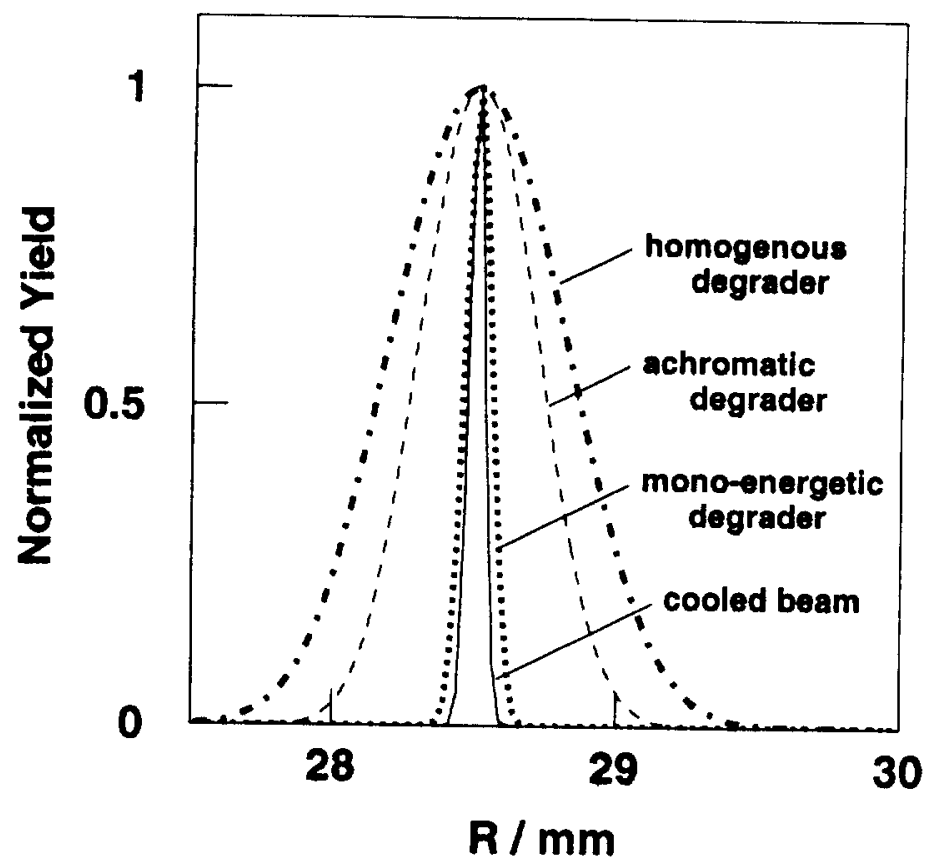

Fig. 5. Range distribution of $640 \mathrm{~A} \cdot \mathrm{MeV}{ }^{215} \mathrm{~Pb}$ in silicon. Depending on the energy spread of the incident fragment beam the range straggling is quite different, but with a specially shaped degrader, a so-called 'monoenergetic degrader', the range profile can be obtained which is quite similar to that obtained with a cooled beam.
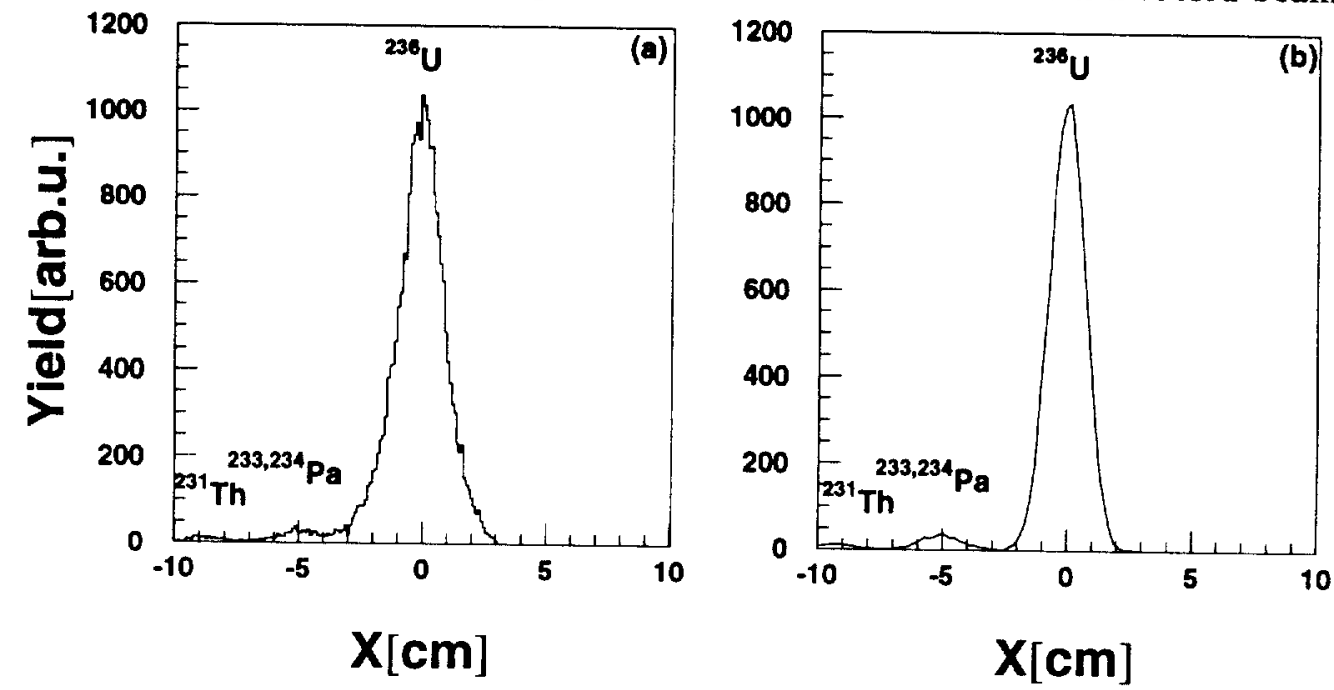

Fig. 6. A stringent test for the performance of the fragment separator FRS and the simulation code MOCADI is the separation of the heaviest fragments. The measured position spectra at the final focus of the FRS (left) clearly show that the heaviest fragments can be spatially separated and that perfect agreement with the experimental results is achieved (right). 


\section{References}

[1] M. Berz, Nucl. Instr. Meth. A 298, 426 (1990).

[2] H. Wollnik, Manual for GICO, Univ. of Gießen (1990).

[3] B. Franczak, Proc. of the Europhysics Conference on Computing in Accelerator Design and Operation, Berlin (1983).

[4] K. L. Brown, Advances Particle Physics 1, 71 (1967).

[5] Th. Schwab, PhD thesis, Univ. of Gießen (1990).

[6] H. Geissel et al., Nucl. Instr. Meth. A282, 247 (1989).

[7] K.-H. Schmidt et al., Nucl. Instr. Meth. A260, 287 (1987).

[8] D. Bazin and B. Sherrill, Phys. Rev. E 50, 4017 (1994).

[9] J. A. Winger et al., Nucl. Instr. Meth. B70, 380 (1992).

[10] H. Geissel et al., Nucl. Instr. Meth. B70, 286 (1992).

[11] B. Franzke et al., Nucl. Instr. Meth. B24/25, 18 (1987).

[12] C. A. Bertulani, G. Baur, Phys. Rep. 163, 299 (1988).

[13] K. Sümmerer et al., Phys. Rev. C42, 2546 (1990).

[14] M. Fauerbach, Diploma Thesis, Internal Report TH Darmstadt (1992).

[15] J. J. Gaimard and K.-H. Schmidt, Nucl. Phys. A531, 709 (1991).

[16] M. Weber et al., Z. Phys. A343, 67 (1992).

[17] S. Kox et al., Phys. Lett. 159 B, 15 (1985).

[18] S. B. Kaufmann et al., Phys. Rev. C22, 1897 (1980).

[19] A. S. Goldhaber, Phys. Lett. 53B, 306 (1974).

[20] D. J. Morrissey, Phys. Rev. C39, 460 (1989).

[21] M. Bernas et al., Phys. Lett. B 331, 19 (1994).

[22] P. Armbruster et al., Z. Phys. A 355, 191 (1996).

[23] G. Münzenberg, in Handbook of Nuclear Decay Modes, de Gruyter, eds. W. Greiner, D. N. Poenaru, in press.

[24] N. Bohr, Kgl. Dan. Mat.-Fys. Medd. 18, No. 8 (1948).

[25] R. Anholt et al., Phys. Rev. A36, 1586 (1987).

[26] C. Scheidenberger et al., Nucl. Instr. Meth. B90, 36 (1994). 
[27] U. Fano, Ann. Rev. Nucl. Sci. 13, 1 (1963).

[28] C. Scheidenberger et al., Phys. Rev. Lett. 73, 50 (1994).

[29] J. Lindhard, A. H. Sørensen, Phys. Rev. A 53, 2443 (1996).

[30] C. Scheidenberger et al., Phys. Rev. Lett. 77, 3987 (1996).

[31] P. Sigmund, Nucl. Instr. Meth. B69, 113 (1992).

[32] V. L. Highland, Nucl. Instr. Meth. 161, 171 (1979).

[33] H. Wollnik, Optics of Charged Particles, Academic Press, New York (1987).

[34] J. P. Dufour et al, Z. Phys. A324, 487 (1986).

[35] A. Magel et al., Nucl. Instr. Meth. B 94, 548 (1994).

[36] H. Geissel et al., contribution to this conference. 